\title{
Near real-time landslide monitoring with the smart soil particles
}

\author{
Ghee Leng Ooi ${ }^{\text {i) }}$, Pin Siang Tan ${ }^{\text {ii) }}$, Meei-Ling Lin ${ }^{\text {iii) }}$, Kuo-Lung Wang ${ }^{\text {iv) }}$, Qian Zhang ${ }^{\text {v) }}$ and Yu-Hsing Wang ${ }^{\text {vi) }}$ \\ i) Ph.D Student, Department of Civil and Environmental Engineering, the Hong Kong University of Science and Technology, Clear \\ Water Bay Rd, Kowloon, Hong Kong. \\ ii) M.Phil. Student, Department of Civil and Environmental Engineering, the Hong Kong University of Science and Technology, Clear \\ Water Bay Rd, Kowloon, Hong Kong. \\ iii) Professor, Department of Civil Engineering, National Taiwan University, 1, Sec 4, Roosevelt Rd, Da'an District, Taipei City, Taiwan. \\ iv) Professor, Department of Civil Engineering, National Chi Nan University, 1, Daxue Road, Puli Township, Nantou County, Taiwan. \\ v) Professor, Department of Computer Science and Engineering, the Hong Kong University of Science and Technology, Clear Water \\ Bay Rd, Kowloon, Hong Kong. \\ vi) Professor, Department of Civil and Environmental Engineering, the Hong Kong University of Science and Technology, Clear Water \\ Bay Rd, Kowloon, Hong Kong.
}

\begin{abstract}
As global warming brings forth extreme precipitation in the typhoon-prone region in Asia, e.g. Hong Kong, Taiwan and Japan, the need for a scalable and yet accurate landslide sensing system becomes increasingly urgent. Natural slopes are the focus since their failures are often large-scale and disastrous; however, it is extremely expensive to monitor every single hazardous natural slope in existence with the current monitoring system. The MEMS (Micro-Electro-Mechanical Systems) technology which makes miniature yet accurate sensors available (e.g. accelerometer, gyroscope and magnetometer), will be pivotal towards the realization of a scalable landslide warning system. In this paper, we present the Smart Soil Particle (SSP) sensing system we build to detect the precursory tilts of a large-scale potential landslide site in LuShan, Taiwan. The SSP consists of the sensing module (i.e., low-noise MEMS accelerometer and a motion sensor) and the processing module (Raspberry Pi, a single-board computer). Both cost less than HKD $\$ 850$; the price makes it possible to deploy a huge amount of SSPs on the slope surface without the fear of losing any. The processing module is capable of handling a large programmable data transfer rate (up to $800 \mathrm{samples} / \mathrm{second} / \mathrm{channel}$ ) through a $3 \mathrm{G}$ modem. In the back-end is a decentralized structured data storage system constructed to handle the massive data inflow from multiple SSPs. The whole system is built to send and receive data at a near real-time speed. With a proper noise control and shielding of the wires, we show that the low-noise SSPs are able to capture a small earthquake and also tiny vibrations due to cars passing by.
\end{abstract}

Keywords: landslide monitoring, early warning system, MEMS, big data

\section{INTRODUCTION}

In Hong Kong, the citizens are generally insensitive to the landslide early warnings issued because they are not specific to where, when and how a landslide is going to occur. These warnings are based on meteorological indicators, e.g., a rainfall amount exceeding a certain threshold value (e.g., see Glade et al. 2000; Chen et al. 2005). Although the rainfall amounts are monitored spatiotemporally, we still need more real-time monitoring data from various instrumentations for an educated judgment to tell where and when a landslide will be. In fact, there are two major reasons for the shortcomings of current landslide monitoring system: (1) expensive instrumentations for field monitoring, and (2) inability to store and process the large amount of monitoring data collected spatiotemporally. In addition, we are facing the difficulty of establishing confidence in the quality of our knowledge collected in the past given the loss of stationarity associated with extreme precipitation events as a result of climate changes (Milly et al. 2008; Dozier and Gail 2009; Westra et al. 2013). Similar hurdles are present in various kinds of civil and environmental engineering monitoring.

Landslide monitoring is a costly endeavor attributed to expensive instruments and manpower, especially when it requires a real-time data for quick decision making (e.g., Wieczorek and Snyder 2009). Instruments currently used to monitor movement data are geophones, ultrasonic sensors and DGPS (differential ground positioning system), which require meticulous fabrication and calibration because of error prone manufacturing lines for anything not produced batch-wise (Cochran et al. 2009). To install these 
expensive sensors on-site would require them to be retrievable after use and need extreme care in handling, necessitating either full-time professional technicians, or an extravagant outdoor package offered by the company.

To solve the issue of expensive sensors, we decide to use the Micro-Electro-Mechanical-Systems (MEMS) sensors. MEMS is a classification of devices, as well as the means of fabrication and manufacturing (Chollet and Liu 2012). The MEMS technology allows batch-wise etching production, thereby minimizing manufacturing cost and eliminating the need for a stringent quality control. Today, MEMS sensors are ubiquitous, found everywhere in smartphones and household devices like the refrigerator. With such cheap and yet accurate sensors at our disposable, we seek to realize the "Smart Dust" concept, which intends to deploy a large amount of cheap, tiny and wireless "motes" that are smart enough to self-organize into a network, allowing a prompt action once a distress signal is detected (Steel 2005). The low cost of each SSP mote (HKD\$750 850/piece in contrast to HKD $\$ 20,000 /$ piece for geophone) allows massive areal deployment and thus obliterates the concern of losing any one of the sensor.

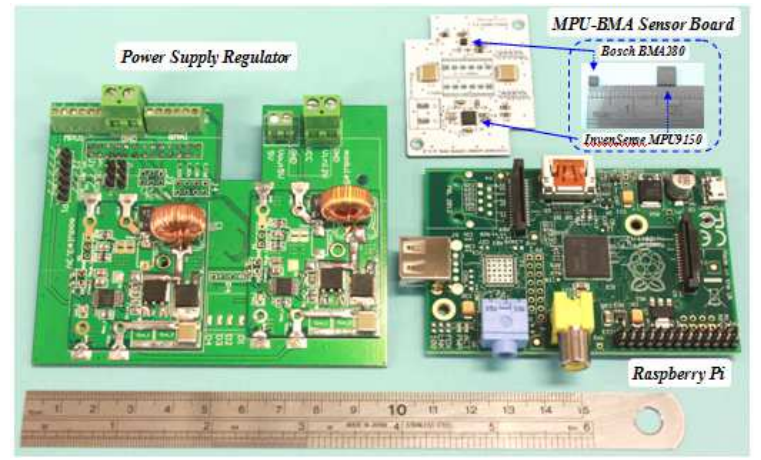

Fig. 1. Sensor and processing module of the Smart Soil Particle.

\section{SMART SOIL PARTICLE}

\subsection{The Sensor and Control Module}

The outcome is the Smart Soil Particle (SSP), as shown in Fig. 1. The SSP comprises: (1) the sensor module, and (2) the control module. The modular design allows individual components (e.g. sensors and processing unit) to be added, changed or replaced as the technologies evolve. Currently, the sensor module allows two MEMS sensors to be mounted on the same board. We have installed various combinations of the following MEMS sensors: (1) ADXL345 (Analog Devices) 3-axis digital accelerometer; (2) BMA280 (Bosch) low-noise 3-axis digital accelerometer; (3) MPU6050 (InvenSense) 3-in-one 3-axis accelerometer, gyroscope and temperature sensor, and (4) MPU9150 (InvenSense) 3-in-one 3-axis accelerometer, gyroscope, magnetometer and temperature sensor. The MEMS accelerometer also can function as a tilt sensor: its zero gravity direct current (DC) offset is designed for the purpose of recording a gravity vector. This yields a good reference for its current state of tilt when the sensor rotates at a quasi-static state (Ooi et al. 2014).

The control module consists of (1) the power supply regulator which stabilizes and minimizes the noise from power source, and (2) the Raspberry $\mathrm{Pi}$, a credit-card sized mini computer which processes and sends the data via a $3 \mathrm{G}$ modem. Each channel of the MEMS sensors in SSP samples at $500 \mathrm{~Hz}$ (there are 14 channels in total, which amounts to $14 \mathrm{kB} / \mathrm{s}$, or 1.21 GB/day). The sampling rate can be programmed on-the-fly, and the maximum capacity is 800 samples/second/channel.

Together with our Taiwan partners from the National Taiwan University and National Taiwan Chi Nan University, we are monitoring a deep-seated huge landslide in LuShan, Taiwan. Currently, there are 3 SSPs running in LuShan, all installed on the slope retaining walls by the roadside where cracks of large landslip surface are observed (see Fig. $2 \& 3$ ). The total data sample per day now tallies at 1.9 billion (or $\sim 32$ GB), and we are receiving such a huge data every single day in real-time.

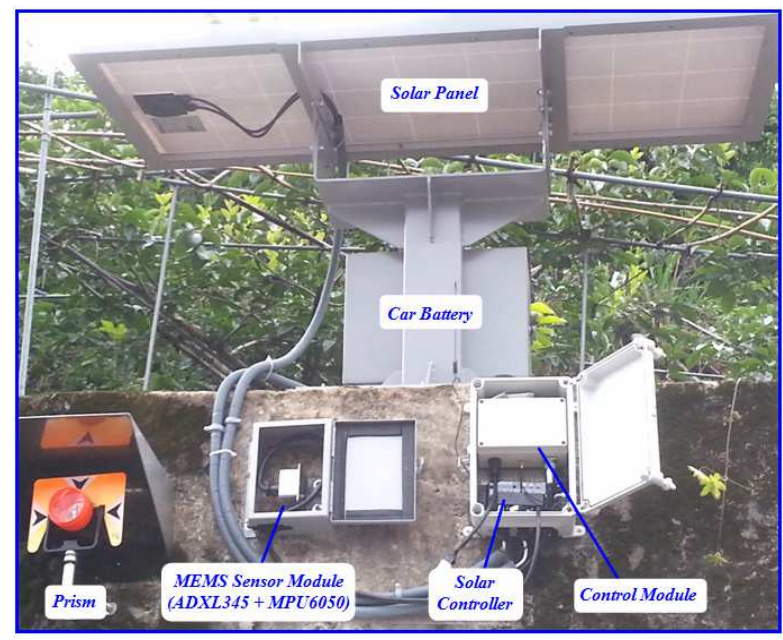

Fig. 2. One of the SSPs installed at the Taiwan LuShan massive deep-seated landslide site.

\subsection{The Data Storage and Processing Architecture}

After creating the field SSP, we built a system that can handle the massive amount of data throughput with MQTT Broker and nodeJS applications. The data are then stored in a 5-node Cassandra cluster, which is a distributed database cluster. The MQTT (Message Queue Telemetry Transport) broker ensures that no matter how many more SSPs are added in the field in the future, the data throughput is scalable. Also, user query can be returned directly from the Cassandra cluster in near-real time for data visualization. This huge and fast data processing we implemented follows the MapReduce architecture and programming model 
pioneered by Google a decade ago (Dean and Ghemawat 2008). The essence of the high performance of MapReduce lies in its simplified programming model, which provides efficient and automatic distribution of data and jobs across machines by scheduling the processes in proximity to the location of data using the information from the distributed file system (Cassandra). This strategy ensures that most of the data is read from the local disk directly into the CPU, this effectively alleviates strain on the network bandwidth and prevents unnecessary network transfers.

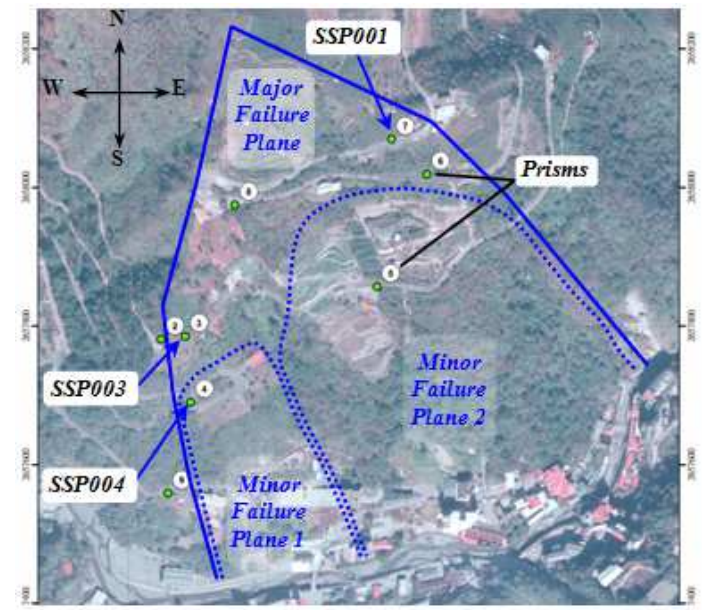

Fig. 3. Plan view of all the failure planes and installation points of the SSPs in LuShan, Taiwan.

\section{PRELIMINARY DATA}

We have built a laboratory flume tailored to examine the initiation mechanisms of flow landslides in response to different hydrologic triggers. We have characterized the initiation features of the landslide body in terms of its surficial and internal local soil movement with our small lab SSPs, i.e., the MEMS accelerometer-based sensor (see Ooi et al. 2010 \& 2014; Ooi and Wang 2014). The lab SSPs installed in the laboratory slope successfully captured various features of the slow and episodic landsliding behavior prior to full fluidization such as (1) local acceleration of the shear zone in the late stage when the soil mass disintegrated with each piece flowing independently; (2) initially rotation-dominant features of the shear zone that became translation-dominant in a later stage; and (3) evolution of soil porosity in response to the rate of the hydrologic trigger.

For the field SSPs, many interesting observations have been recorded. For example, we had discovered motions recorded during Typhoon Matmo on 23-24 July 2014 and also an earthquake vibration recorded on $16^{\text {th }}$ July 2013. The data recorded during this earthquake and tiny vibrations due to cars passing by are presented in following sessions.

\subsection{Small Earthquake}

On July 16, 2013, a Richter magnitude 5.5 earthquake (longitude: 121.5 ${ }^{\circ}$; latitude: 24.28 ${ }^{\circ}$ ) centered in Hualian County, Xiulin township hit northern Taiwan. The epicenter was very close to LuShan, and a check on the seismograph in the Puli township (longitude: $120.96^{\circ}$; latitude: $24.01^{\circ}$ ) nearby showed a peak acceleration of $4.85 \mathrm{gal}\left(\mathrm{cm} / \mathrm{s}^{2}\right)$ in the vertical axis (see Fig. 3). Here we show the same time extract from our SSP001 (longitude: $121.18^{\circ}$; latitude: $24.03^{\circ}$ ) as the Puli seismograph. Note that SSP001 is situated near the crown of the LuShan slip plane. We can see that all the peak accelerations in the vertical and North-South (NS) axes were amplified in the LuShan slip zone. The maximum vibration occurred in the NS direction, which turns out to be dominating slip direction of LuShan, which is to the south.

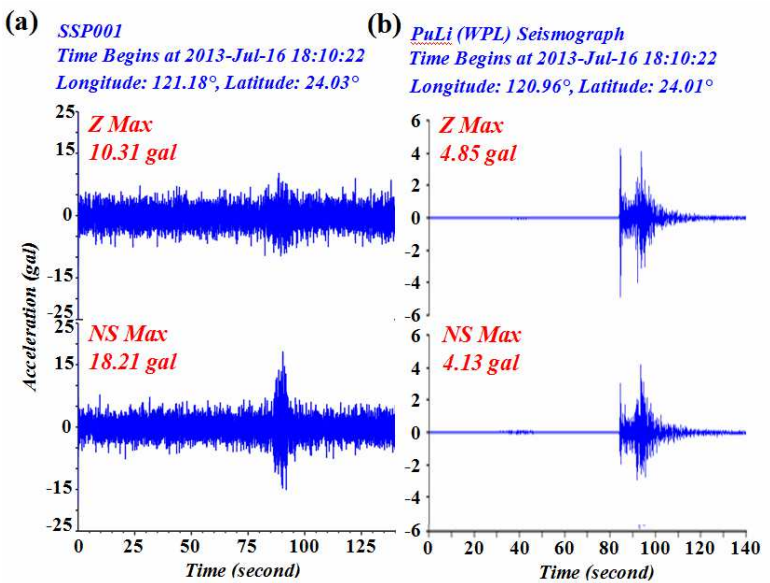

Fig. 4. Vibration due to a small earthquake as measured by: (a) SSP001 situated near the crown of the LuShan slip plane, and (b) the seismograph at PuLi township.

\subsection{Car Passing By}

Through a proper noise control by tuning the capacitance and resistance of the power supply regular and also proper shielding of the wires, we can minimize the noise level of the SSPs. The accelerometers can capture the slight vibrations caused by cars when they pass by the retaining wall. In Fig. 4 we show that there are three cars passing by the retaining wall in sequence near SSP001 at LuShan, Taiwan; and in Fig. 5 we show the corresponding vibrations captured by the SSP installed on the retaining wall.

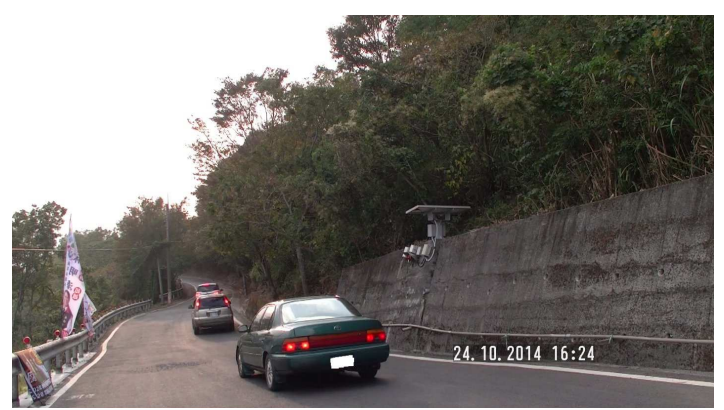

Fig. 5. Three cars passing by consecutively near SSP001 at LuShan, Taiwan. 

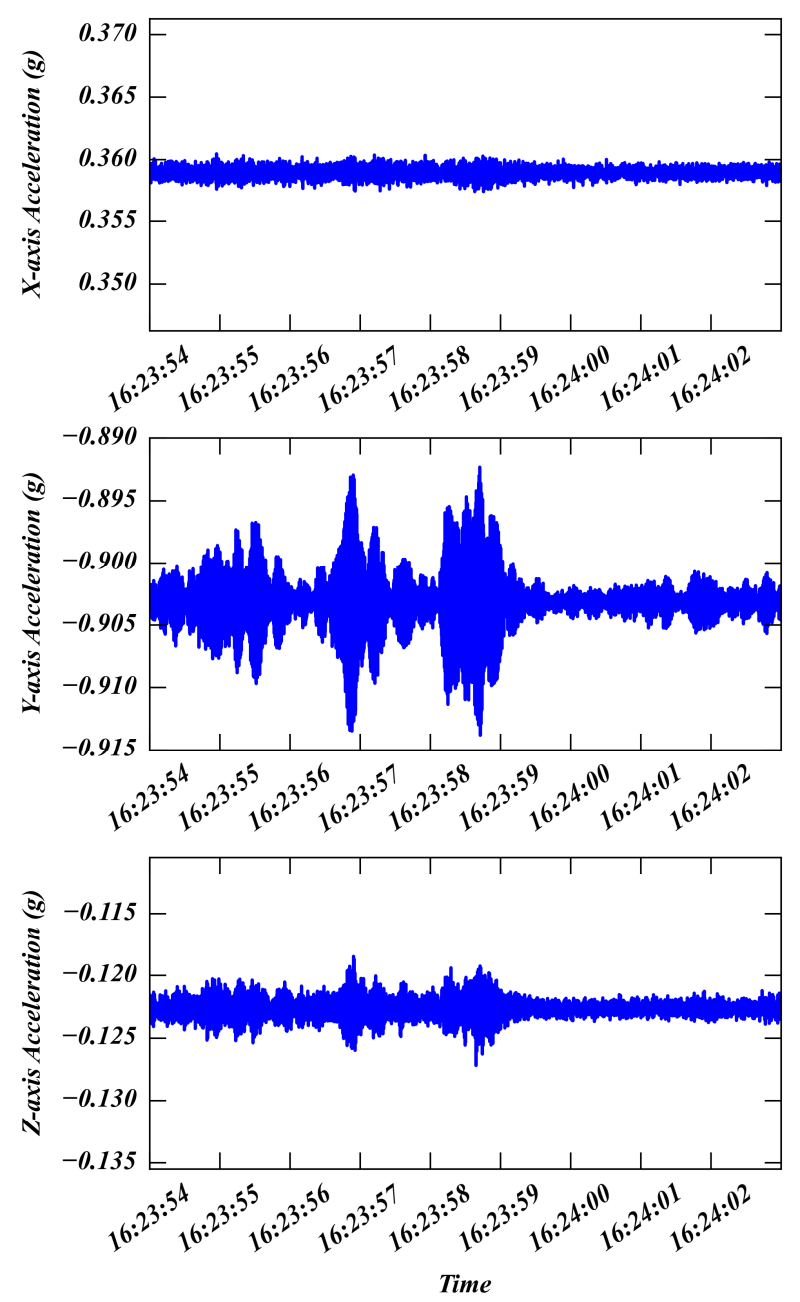

Fig. 6. Corresponding vibrations from the three vehicles captured by the SSP

\section{SUMMARY AND CONCLUSIONS}

The SSPs we designed are economical and easy to deploy in the field. Built from the increasingly small and low-noise MEMS sensors (accelerometers, gyroscope, magnetometer and temperature sensor) available today, massive deployment of the SSPs allow us to monitor site movements in near-real time without the fear of losing any of them in the event of a landslide or debris flow. Here we suggest that quantity of the economical yet sensitive MEMS motion sensors deployed on the slope surface will compensate for the need to deploy expensive field observation method such as the piezometer. The flexibility also allows the SSPs to be installed in recent debris flow sites where the site conditions are harsh and installation works might be arduous. With a proper noise control and shielding of the wires, we have shown that the SSPs can record a small earthquake and a car passing by, and we are confident that small precursory tilts of the slope as it moves can also be measured. Currently, we are building the SSP and its ancillary data storage and processing architecture towards a large scale, economical landslide early warning and monitoring system.

\section{ACKNOWLEDGEMENTS}

This research was supported by the Hong Kong Innovation and Technology Fund (ITS/142/13) and Research Grants Council (HKUST9/CRF/09 and HKUST6/CRF/12R).

\section{REFERENCES}

1) Chen, C. Y., Chen, T. C., Yu, F. C., Yu, W. H., and Tseng C.C. (2005): Rainfall Duration and Debris-Flow Initiated Studies for Real-Time Monitoring, Environ. Geol., 47, 715-724.

2) Chollet, F., and Liu, H. (2012): A (not so) short introduction to Micro Electro Mechanical Systems, MEMScyclopedia.org.

3) Cochran, E., Lawrence, J., Christensen, C. and Chung, A. (2009): A novel strong-motion seismic network for community participation in earthquake monitoring, IEEE Instrumentation \& Measurement Magazine, 12(6), 8-15.

4) Dean, J., and Ghemawat, S. (2008): MapReduce: simplified data processing on large clusters, Communications of the ACM, 51(1), 107-113.

5) Dozier, J., and Gail, W.B. (2009): The emerging science of environmental applications, The fourth paradigm: data-intensive scientific discovery, 13-19.

6) Glade T, Crozier M, Smith P (2000): Applying probability determination to refine landslide-triggering rainfall thresholds using an empirical "antecedent daily rainfall model", Pure Appl Geophys, 157, 1059-1079.

7) Milly, P.C.D., Betancourt, J., Falkenmark, M., Hirsch, R.M., Kundezewicz, Z.W., Lettenmaier, D.P., and Stouffer, R.J. (2008): Stationarity is dead: whither water management? Science, 319, 573-574.

8) Ooi, G.L. and Wang, Y.H. (2014): Applying MEMS accelerometers to measure ground vibration and to characterize landslide initiation features in laboratory flume test, Geo-Congress 2014, Geo-Characterization and Modeling for Sustainability, Atlanta.

9) Ooi, G.L., Wang, Y.H., Wong, T.W., and Wong, C.S. (2010): An exploratory study on the application of miniature sensors in monitoring landslide motion, $7^{\text {th }}$ International Conference on Physical Modelling in Geotechnics (ICPMG 2010), ETH Zurich.

10) Ooi, G.L., Wang, Y.H., Tan, P.S., So, C.F., Leung, M.L., Li, X. and Lok, K.H. (2014): An Instrumented Flume to Characterize the Initiation Features of Flow Landslides, Geotechnical Testing Journal, 37(5).

11) Steel, D. (2005): Smart Dust, UH ISRC Technology Briefing, University of Houston, Houston, TX.

12) Westra, S., Alexander, L.V., and Zwiers, F.W. (2013): Global increasing trends in annual maximum daily precipitation, Journal of Climate, American Meteorological Society, 26, 3904-3918.

13) Wieczorek, G.F., and Snyder, J.B. (2009): Monitoring slope movements, Geological Monitoring, The Geological Society of America, ISBN: 978-0813760322. 